\title{
Comportamiento Resistente de Suelos Orgánicos Estabilizados con Tanino
}

\author{
Viviana E. Fabre, Marcela B. Bizzotto y Jirina C. Tirner \\ Universidad Nacional del Nordeste (UNNE), Facultad de Ingeniería, Instituto de Estabilidad, \\ Laboratorio de Suelos, Av. Las Heras 727, (3500) Resistencia, Chaco-Argentina \\ (e-mail: vfabre@ing.unne.edu.ar)
}

\begin{abstract}
Resumen
El objetivo del presente trabajo fue evaluar la resistencia de suelos orgánicos del noreste argentino, una vez cumplido el proceso de estabilización con distintas resinas. Se realizaron ensayos de resistencia triaxial sobre probetas de suelo orgánico, natural y estabilizado. El tratamiento se materializó incorporando al suelo resinas en las que intervinieron productos obtenidos de la cadena de fabricación del tanino de Quebracho Colorado, un aldehído y agua. La concentración de la resina fue variable, como también la proporción con respecto al peso seco del suelo en que se la agregaba y los días de curado. Los ensayos triaxiales permitieron el trazado de las líneas de resistencia intrínseca para evaluar la acción del estabilizante. Los resultados indicaron un incremento en la resistencia mecánica del suelo estabilizado, pudiéndose identificar la resina que indujo a un mejor efecto sobre el suelo natural, permitiendo extender las posibilidades de aplicación del material tratado.
\end{abstract}

Palabras clave: suelo, estabilización, resinas, tanino, resistencia

\section{Strength Behavior of Organic Soils Stabilized with Tannin}

\begin{abstract}
The aim of this work was evaluating the strength of organic soils in northeastern region of Argentina after completing the stabilization process with different types of resins. Several triaxial essays on probes of organic, natural and stabilized soils, were carried out. Treatment was materialized by adding resins to soil, in wich products obtained in the chain of production of tannin from "Quebracho Colorado", an aldehyde and water took part. Concentration of resin was variable, as well as rate of dry weight of soil in wich it was added and the curing time. Tri - axial tests allowed drawing of intrinsic strength lines in order to evaluate the action of stabilizer. Results indicated a rise of mechanical strength of stabilized soil, allowing identification of resin wich induced a better effect on natural soil and to extend possibilities of application of treated material.
\end{abstract}

Keywords: soil, stabilization, resins, tannin, strength 


\section{INTRODUCCIÓN}

Los suelos de la región noreste de la República Argentina usados como vías de comunicación en zonas dedicadas a la explotación agrícola-ganadera son típicamente arcillosos y con contenido de materia orgánica. Al ser utilizados sin un tratamiento adecuado ocasionan dificultades, especialmente ante cambios importantes en el contenido de agua en épocas de intensas precipitaciones.

Por otra parte, desde el punto de vista de la mecánica de suelos, la materia orgánica es un componente no deseado. Su estructura abierta y esponjosa y la debilidad mecánica de sus constituyentes reduce la capacidad de carga del suelo, aumenta su deformabilidad cuando se lo somete a presión o cambios en el contenido de agua y además tiende a dar una reacción ácida al agua del suelo, lo que puede provocar corrosión en los elementos constructivos enterrados en él. Por todo esto es una práctica común, en la medida de las posibilidades, eliminar en obra las capas superficiales de suelos que contienen cantidades apreciables de materia orgánica.

Surge así la necesidad de dar solución a estos problemas para asegurar la transitabilidad de los caminos y sendas interiores de los establecimientos por aplicación de un método de mejoramiento del suelo natural arcilloso y con apreciable contenido de materia orgánica.

La elección del método debe tener en cuenta el tipo de suelo a estabilizar, el uso que se le dará al mismo, la disponibilidad de los materiales, el conocimiento del arte, el aspecto económico, el impacto sobre el medio ambiente, etc. (Garnica Anguas et al., 2002).

Existen distintos métodos de estabilización de los suelos; cada uno de ellos emplea diferentes productos estabilizantes o inclusive la combinación de algunos de ellos: cal, cemento Portland, productos asfálticos, resinas y polímeros, sales, cenizas volantes, aceite sulfonado (Camacho et al., 2006), fibras de polipropileno (Chaosheng et al., 2007), desechos quemados de olivos (Attom y AlSharif, 1998), entre otros. Debe mencionarse que en la actualidad existe un gran número de resinas sintéticas para lograr una mejora en las propiedades ingenieriles de los suelos, entre ellas el fenolformaldehído, la urea-formaldehído, la anilina-furfural, el vinsol, el Copal Damar, la colofonia (rosin) y las resinas furan.

Estabilización de suelos con tanino (Di Rado et al., 2001), comenta las observaciones efectuadas por uno de los autores en el lugar de disposición final de los residuos de una fábrica de tanino obtenido del árbol de Quebracho Colorado (Schinopsis balansae); en el lugar se podía observar un talud casi vertical a pesar del tipo de suelo existente, lo que indicaba una modificación de las características del mismo. Esto fue el origen de una serie de proyectos de investigación; en una primera etapa estuvieron orientados a estudiar el efecto estabilizante de una resina sintética, en la que intervenía el tanino, en las propiedades físicas y mecánicas del suelo para una posterior aplicación vial.

Se dispone de antecedentes referidos a la estabilización de arenas con colofonia y taninos de Terminalia alata (Gomal et al., 1988), pero no fue posible encontrar referencias comprobables de estudios realizados en suelos arcillosos y con contenido de materia orgánica estabilizados con tanino.

Se trabajó con distintos tipos de suelos y con resinas en diferentes composiciones, concentraciones y proporciones con respecto al peso de suelo seco. Todos los estudios iniciales y sus resultados fueron explicados en la publicación antes citada (Di Rado et al., 2001). A manera de síntesis de aquella etapa de trabajo, se presenta más adelante la Tabla 1.

Las experiencias realizadas en laboratorio y en campo confirmaron las hipótesis de trabajo: tanto las características físicas, plásticas y mecánicas del suelo, como su resistencia al agua en procesos de inmersión o abrasivos por goteo, fueron superiores con relación al suelo natural. Además, se corroboró la posibilidad de estabilización de suelos con considerable contenido de materia orgánica, agregando así un nuevo tipo de suelo factible de ser estabilizado con resinas sintéticas. Este aporte a la rama de la ingeniería, marco de este trabajo, dio origen a la obtención de la titularidad de una Patente de Invención (Di Rado et al., 2006). 
Tabla 1: Composición de las resinas

\begin{tabular}{|c|c|c|c|c|c|c|c|}
\hline \multirow[b]{2}{*}{ Resina } & \multicolumn{7}{|c|}{ Componentes } \\
\hline & $\begin{array}{r}\text { Tanino (1 } \\
(\mathrm{mol})\end{array}$ & $+\mathrm{F}$ & $\begin{array}{l}\operatorname{rmol}(F) \\
(\mathrm{mol})\end{array}$ & $\begin{array}{c}\text { Tanino (T) } \\
(\mathrm{N})\end{array}$ & $\begin{array}{l}+ \text { Formol }(F) \\
(N)\end{array}$ & $\begin{array}{c}\text { Tanino }(\mathrm{T})+ \\
(\%)\end{array}$ & $\begin{array}{c}\text { Formol (F) } \\
(\%)\end{array}$ \\
\hline R1 & $1 \mathrm{~T}$ & + & $1 \mathrm{~F}$ & 1.541 & +0.294 & $84.0+$ & +16.0 \\
\hline R2 & $2 T$ & + & $1 \mathrm{~F}$ & 3.082 & +0.294 & 91.3 & $+\quad 8.7$ \\
\hline R3 & $3 \mathrm{~T}$ & + & $1 \mathrm{~F}$ & 4.624 & +0.294 & 94.0 & 6.0 \\
\hline R4 & $4 \mathrm{~T}$ & + & $1 \mathrm{~F}$ & 6.165 & +0.294 & 95.4 & +4.6 \\
\hline R5 & $1 \mathrm{~T}$ & + & $2 \mathrm{~F}$ & 1.541 & +0.588 & 72.4 & +27.6 \\
\hline R6 & $1 \mathrm{~T}$ & + & $3 \mathrm{~F}$ & 1.541 & +0.883 & 63.6 & +36.4 \\
\hline R7 & $1 \mathrm{~T}$ & + & $4 \mathrm{~F}$ & 1.541 & $+\quad 1.177$ & 56.7 & +43.3 \\
\hline
\end{tabular}

El objetivo del presente trabajo fue evaluar el comportamiento mecánico por medio de ensayos triaxiales de suelos orgánicos del noreste argentino estabilizados con resinas compuestas por alguno de los productos derivados de la elaboración del tanino.

Los altos valores de resistencia mecánica alcanzados por las muestras de suelo estabilizado y ensayado, permitieron ampliar el horizonte de aplicación de este nuevo material, de manera tal de utilizarlo tanto en obras viales como en la fabricación de componentes de viviendas.

\section{PROCEDIMIENTO}

\section{Materiales utilizados}

Las muestras de suelo analizadas fueron extraídas de la superficie de un solar ubicado en Villa Don Santiago de la Ciudad de Resistencia, capital de la Provincia del Chaco. Fue denominado SON: Suelo Orgánico Natural (Di Rado et al., 2002). Por aplicación de ensayos bajo Normas ASTM (D2216-05, 2005; ASTM D4318-05, 2005; ASTM D1140-00, 2006; ASTM D2487, 2006; ASTM D2850, 2007; ASTM D421, 2007), se definieron sus características plásticas, las que se muestran en la Tabla 2, como también su clasificación SUCS y HRB. En ella aparece el porcentaje de materia orgánica contenida.

Siguiendo el criterio explicado en Manual of Soil Laboratory Testing (Head, 1986), para no afectar los resultados de los ensayos debido a la presencia de materia orgánica, se utilizó la estufa de secado a una temperatura de $60^{\circ} \mathrm{C}$ en todas las determinaciones.

Tabla 2: Características del suelo orgánico empleado en los ensayos

\begin{tabular}{|c|c|c|c|c|c|c|c|c|}
\hline Suelo & $\begin{array}{c}\text { Pasa } \\
\# 200\end{array}$ & LL & LP & IP & LC & $\begin{array}{c}\text { Materia } \\
\text { orgánica }\end{array}$ & $\begin{array}{c}\text { Clasificación } \\
\text { SUCS }\end{array}$ & $\begin{array}{c}\text { Clasificación } \\
\text { HRB }\end{array}$ \\
\hline SON & $92 \%$ & 61,8 & 34,5 & 27,3 & 30,3 & $7,50 \%$ & OH & A-7-5 (31) \\
\hline
\end{tabular}

La resina empleada fue la denominada $\mathrm{R} 7$ en Tabla 1, elaborada a partir de uno de los productos derivados de la cadena de fabricación del tanino, en un porcentaje del 56,7 \%, al que se adicionó un aldehído en un $43,3 \%$ y agua en cantidad necesaria, según lo previamente resuelto a través del ensayo de compactación (Di Rado et al., 2001). Al ser la resina que menor porcentaje de tanino necesita, resulta la de menor costo. Se la aplicó en una proporción del $10 \%$ del peso seco del suelo (E10). De allí surgió la denominación usada: E10R7.

Para la elaboración de la resina se emplearon tres productos derivados del tanino (Di Rado et al., 2002):

Tupafin: producto final de la fabricación del tanino. Utilizado en el estado en que fue provisto por la fábrica, es decir, un polvo de granulometría muy fina logrado por un proceso de atomización. A las 
mezclas de suelo natural tratadas con la resina en la que interviene este producto se las denomina SONTUP.

QS: resina natural 100\% tanino vegetal. Su presentación en grumos de gran tamaño hizo necesario su paso por el molino de bolas. Estas mezclas se conocen como SONQS.

Extracto natural: producto obtenido en la etapa final de elaboración del tanino en bloque. Debido a su presentación en forma de líquido viscoso, se lo sometió a un sencillo tratamiento consistente en secado a $60^{\circ} \mathrm{C}$ hasta transformarlo en sólido, luego se realizó el molido del material y el posterior tamizado por el Tamiz $\mathrm{N}^{\circ}$ 40, para permitir un buen mezclado con el suelo. A estas mezclas se las nombra como SONPEN (Suelo Orgánico Natural + Polvo de Extracto Natural).

El aldehído utilizado fue formol analítico.

\section{Metodología}

Elegidos los materiales, se conformaron series de probetas para ser ensayadas a compresión confinada, siguiendo el procedimiento correspondiente al Ensayo Triaxial No Consolidado - No Drenado (UU: Unconsolidated - Undrained) para suelos cohesivos (ASTM D2850, 2007), entendiendo que este tipo de ensayo es el adecuado para obtener datos ciertos con respecto a la resistencia al corte del material a humedad constante.

Se prepararon probetas de suelo natural y de cada uno de los tratamientos realizados siguiendo el procedimiento del ensayo de Compactación Miniatura Harvard (con una energía de 50 aplicaciones por cada capa, 5 capas en total y utilizando el resorte de 18 libras). Fueron denominadas genéricamente SON, SONTUP, SONQS y SONPEN, como se ha expuesto anteriormente. Como mínimo se prepararon tres probetas por cada presión de cámara a utilizar y por cada tiempo de curado.

Se las sometió a curado en recipientes adecuados a tal fin, completamente herméticos, por la siguiente cantidad de días: 7, 14, 21 y 28 (en adelante: 7 DC, 14 DC, 21 DC y 28 DC).

Luego de cumplirse los periodos de curado establecidos, se sometió a las probetas al ensayo de compresión triaxial con las siguientes presiones de cámara $(\sigma 3): 50,100$ y $200 \mathrm{kPa}$. Una vez realizados los ensayos triaxiales, se evaluaron los resultados y se trazaron los círculos de Mohr para las distintas presiones de cámara, en un sistema de ejes $\sigma$ (tensión normal) $-\tau$ (tensión tangencial).

Se consideró, para la construcción de los respectivos círculos de Mohr, trabajar con el promedio de los valores correspondientes a las probetas ensayadas bajo cada una de las tres presiones de cámara. En consecuencia, una vez trazados los círculos se realizaba una comparación entre ellos, para cada $\sigma 3$, tomando el valor promedio si la diferencia entre ellos era suficientemente aceptable. De no ser así, se repetía el ensayo cuestionado.

A continuación se dibujaron todas las envolventes de Mohr a los fines de hacer las consideraciones correspondientes y las comparaciones entre el suelo natural y el suelo tratado con cada una de las resinas previstas.

Finalmente, para el trazado de dichas envolventes se utilizó un software CAD, estableciendo los puntos de tangencia de cada círculo y suponiendo un arco de circunferencia. Dada la proximidad entre ellos, el error que se comete puede ser admisible. La envolvente determinada por estos tres puntos de coordenadas definidas, se trazó según una curva potencial del tipo y $=$ a. $X^{P}$. En esta expresión:

$\mathrm{y}$ : tensiones tangenciales

$\mathrm{x}$ : tensiones normales

a: constante para cada curva

$P$ : valor de la potencia. 


\section{RESULTADOS Y DISCUSIONES}

Se muestran a continuación, en Tabla 3 y Figura 1, las características resistentes de SON para las tres presiones de cámara preestablecidas.

A continuación, en Tablas 4 a 6 , se pueden observar los resultados obtenidos para el suelo tratado con cada una de las resinas, para las mismas presiones de cámara y respetando los periodos de curado previamente definidos.

Tabla 3: SON. Promedio de tres Círculos de Mohr por cada presión de cámara

\begin{tabular}{|c|c|c|c|c|c|}
\hline \multirow{2}{*}{ Ensayos } & \multicolumn{5}{|c|}{ Presiones Promedio (kPa) } \\
\cline { 2 - 6 } & $\begin{array}{c}\text { Presión } \\
\text { cámara }\end{array}$ & $\begin{array}{c}\text { Esf. } \\
\text { desviador }\end{array}$ & Axial (máx) & $\begin{array}{c}\text { Centro Círculo } \\
\text { Mohr }\end{array}$ & $\begin{array}{c}\text { Tangencial } \\
\text { (máx) }\end{array}$ \\
\cline { 2 - 6 } & $\sigma_{3}$ & $p=\sigma_{1}-\sigma_{3}$ & $\sigma_{1}=\sigma_{3}+p$ & $\sigma=\left(\sigma_{1}+\sigma_{3}\right) / 2$ & $\tau=\left(\sigma_{1}-\sigma_{3}\right) / 2$ \\
\hline $1^{\circ}$ & 50 & 440 & 490 & 270 & 220 \\
\hline $2^{\circ}$ & 100 & 530 & 630 & 365 & 265 \\
\hline $3^{\circ}$ & 200 & 590 & 790 & 495 & 295 \\
\hline
\end{tabular}

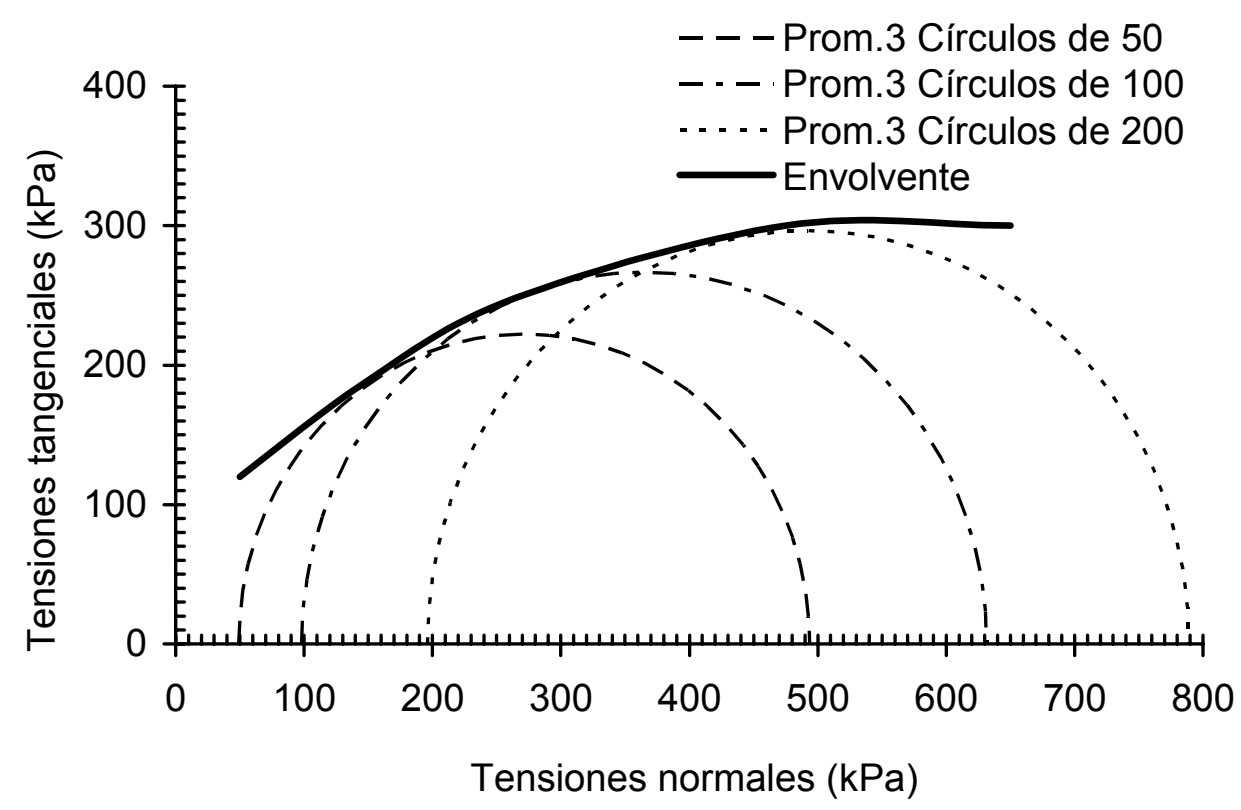

Fig. 1: Círculos de Mohr y Envolvente de Mohr para SON.

Consideraciones sobre el tiempo de curado para cada resina utilizada

Las Tablas 4 a 6 muestran claramente el efecto beneficioso que ejerce sobre el suelo natural cualquiera de los tipos de resina utilizados, lo que se representa también en las Figuras 2 a 4.

Es posible cuantificar la mejora, advirtiéndose que en el caso de la mezcla SONPEN se pasa de un incremento, en promedio, del $57 \%$ en los resultados obtenidos a los 7 DC a un incremento, en promedio, igual al 100\% para el mayor tiempo de curado (28 DC). Si se estudia la mezcla SONQS, la mejora en los valores obtenidos va de un $14 \%$ a un $48 \%$ a iguales periodos de curado, notablemente menor a la primera mezcla mencionada. Finalmente, se analizan las magnitudes obtenidas en los ensayos realizados sobre probetas de mezcla SONTUP, dichos aumentos se ubican en un $108 \%$ a 7 DC y alcanzan un $150 \%$ a los 28 DC. 
En todos los casos se hace evidente que, usando la misma resina, con período de curado creciente, aumenta el efecto estabilizante respecto del suelo sin tratar.

Tabla 4: SONPEN. Promedio de tres Círculos de Mohr por cada presión de cámara y para los distintos DC.

\begin{tabular}{|c|c|c|c|c|c|c|c|c|c|}
\hline \multirow{3}{*}{ Ensayos } & \multirow{3}{*}{$\begin{array}{c}\text { Presión } \\
\text { de } \\
\text { cámara } \\
(\mathrm{kPa})\end{array}$} & \multicolumn{8}{|c|}{ Presiones Promedio (kPa) } \\
\hline & & \multicolumn{2}{|c|}{$7 \mathrm{DC}$} & \multicolumn{2}{|c|}{$14 \mathrm{DC}$} & \multicolumn{2}{|c|}{$21 \mathrm{DC}$} & \multicolumn{2}{|c|}{$28 \mathrm{DC}$} \\
\hline & & $\sigma_{1}$ & $\tau$ & $\sigma_{1}$ & $\tau$ & $\sigma_{1}$ & $\tau$ & $\sigma_{1}$ & $\tau$ \\
\hline $1^{\circ}$ & 50 & 750 & 350 & 880 & 415 & 970 & 460 & 920 & 435 \\
\hline $2^{\circ}$ & 100 & 920 & 410 & 1060 & 480 & 1190 & 545 & 1140 & 520 \\
\hline $3^{\circ}$ & 200 & 1130 & 465 & 1230 & 515 & 1440 & 620 & 1430 & 615 \\
\hline
\end{tabular}

Tabla 5: SONQS. Promedio de tres Círculos de Mohr por cada presión de cámara y para los distintos DC.

\begin{tabular}{|c|c|c|c|c|c|c|c|c|c|}
\hline \multirow{2}{*}{ Ensayos } & $\begin{array}{c}\text { Presión } \\
\text { de } \\
\text { cámara } \\
(\mathrm{kPa})\end{array}$ & \multicolumn{9}{|c|}{$7 \mathrm{DC}$} & \multicolumn{2}{|c|}{$14 \mathrm{DC}$} & \multicolumn{2}{|c|}{$21 \mathrm{DC}$} & \multicolumn{2}{|c|}{$28 \mathrm{DC}$} \\
\cline { 2 - 11 } & $\sigma_{3}$ & $\sigma_{1}$ & $\tau$ & $\sigma_{1}$ & $\tau$ & $\sigma_{1}$ & $\tau$ & $\sigma_{1}$ & $\tau$ \\
\hline $1^{\circ}$ & 50 & 540 & 245 & 630 & 290 & 680 & 315 & 720 & 335 \\
\hline $2^{\circ}$ & 100 & 670 & 285 & 770 & 335 & 800 & 350 & 870 & 385 \\
\hline $3^{\circ}$ & 200 & 930 & 365 & 990 & 395 & 1040 & 420 & 1070 & 435 \\
\hline
\end{tabular}

Tabla 6: SONTUP. Promedio de tres Círculos de Mohr por cada presión de cámara y para los distintos DC.

\begin{tabular}{|c|c|c|c|c|c|c|c|c|c|}
\hline \multirow{2}{*}{ Ensayos } & \multirow{2}{*}{$\begin{array}{c}\text { Presión } \\
\text { de } \\
\text { cámara } \\
(\mathrm{kPa})\end{array}$} & \multicolumn{2}{|c|}{$7 \mathrm{DC}$} & \multicolumn{2}{|c|}{$14 \mathrm{DC}$} & \multicolumn{2}{|c|}{$21 \mathrm{DC}$} & \multicolumn{2}{|c|}{$28 \mathrm{DC}$} \\
\cline { 3 - 11 } & $\sigma_{3}$ & $\sigma_{1}$ & $\tau$ & $\sigma_{1}$ & $\tau$ & $\sigma_{1}$ & $\tau$ & $\sigma_{1}$ & $\tau$ \\
\hline $1^{\circ}$ & 50 & 960 & 455 & 1110 & 530 & 1190 & 570 & 1240 & 595 \\
\hline $2^{\circ}$ & 100 & 1150 & 525 & 1280 & 590 & 1490 & 695 & 1440 & 670 \\
\hline $3^{\circ}$ & 200 & 1500 & 650 & 1580 & 690 & 1740 & 770 & 1720 & 760 \\
\hline
\end{tabular}

Se representa a continuación, en las Figuras 2 a 4, el trazado de las líneas de resistencia intrínseca para cada mezcla suelo - resina e indicando los resultados obtenidos para cada periodo de curado.

\section{SONPEN}

La Figura 2 pone de manifiesto los mejores resultados analizados en la Tabla 4 y logrados al permitir el transcurso de mayor cantidad de días de curado. El incremento es notable a partir de los 7 DC hasta cumplidos los $21 \mathrm{DC}$, a partir del cual los resultados vuelven prácticamente a repetirse para 28 DC.

\section{SONQS}

La influencia de la incorporación de esta resina puede apreciarse observando los valores alcanzados en la Tabla 5 y la configuración adoptada por las líneas envolventes de la Figura 3. Con esta mezcla se puede apreciar menor ganancia de resistencia, para cada uno de los períodos de curado, en relación a los obtenidos con SONPEN. 


\section{SONTUP}

Los resultados están agrupados en la Tabla 6 y en la Figura 4. Observando esta figura se advierte claramente la influencia de 7 DC para la mejora mecánica del suelo natural, que se mantiene sostenidamente hasta cumplir 21 DC. A partir de estos días de curado y hasta 28 DC, no muestra variaciones de relevancia, manteniéndose los valores hallados en el mismo orden de importancia.

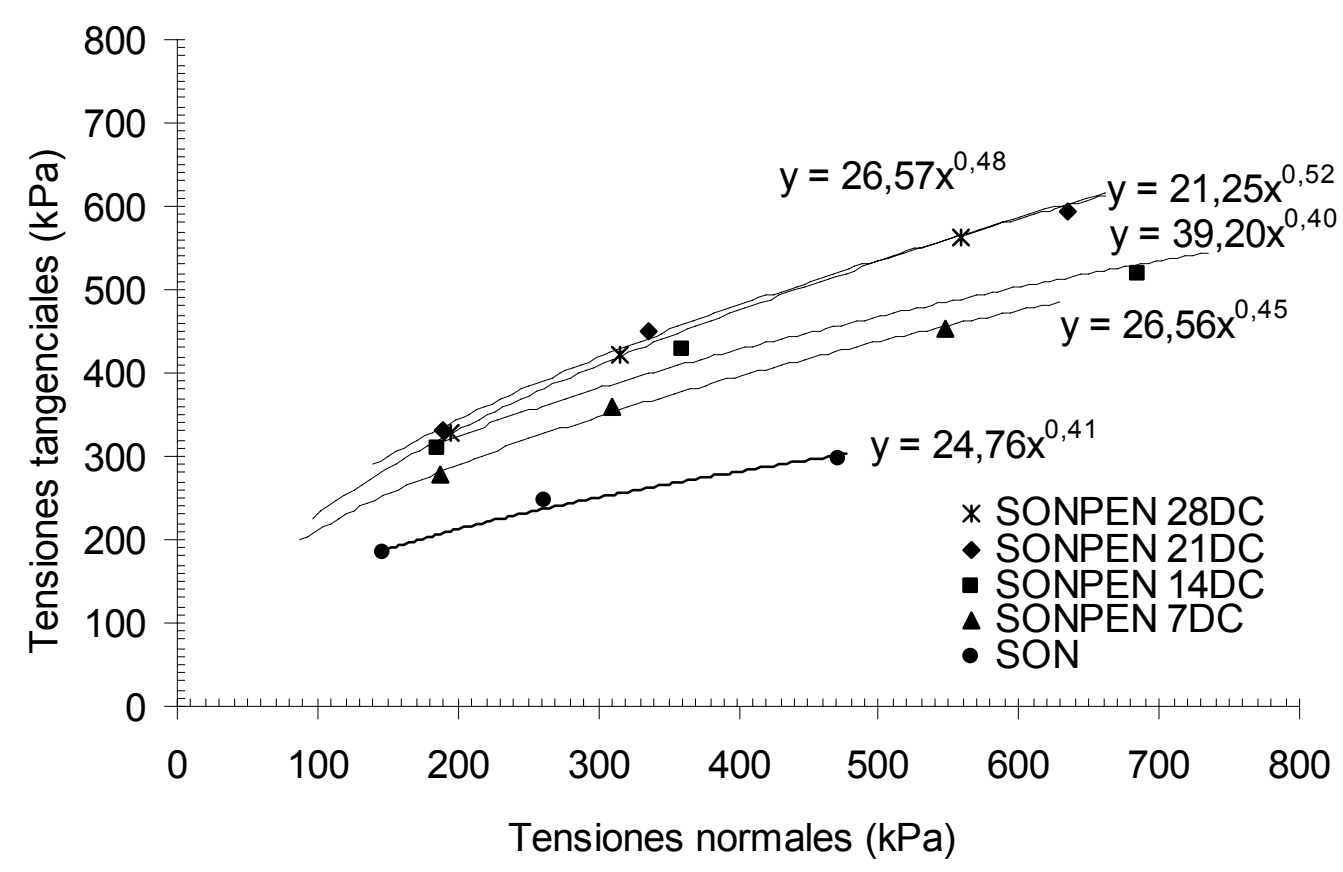

Fig. 2: Envolventes de Mohr para cada período de curado - SONPEN y SON.

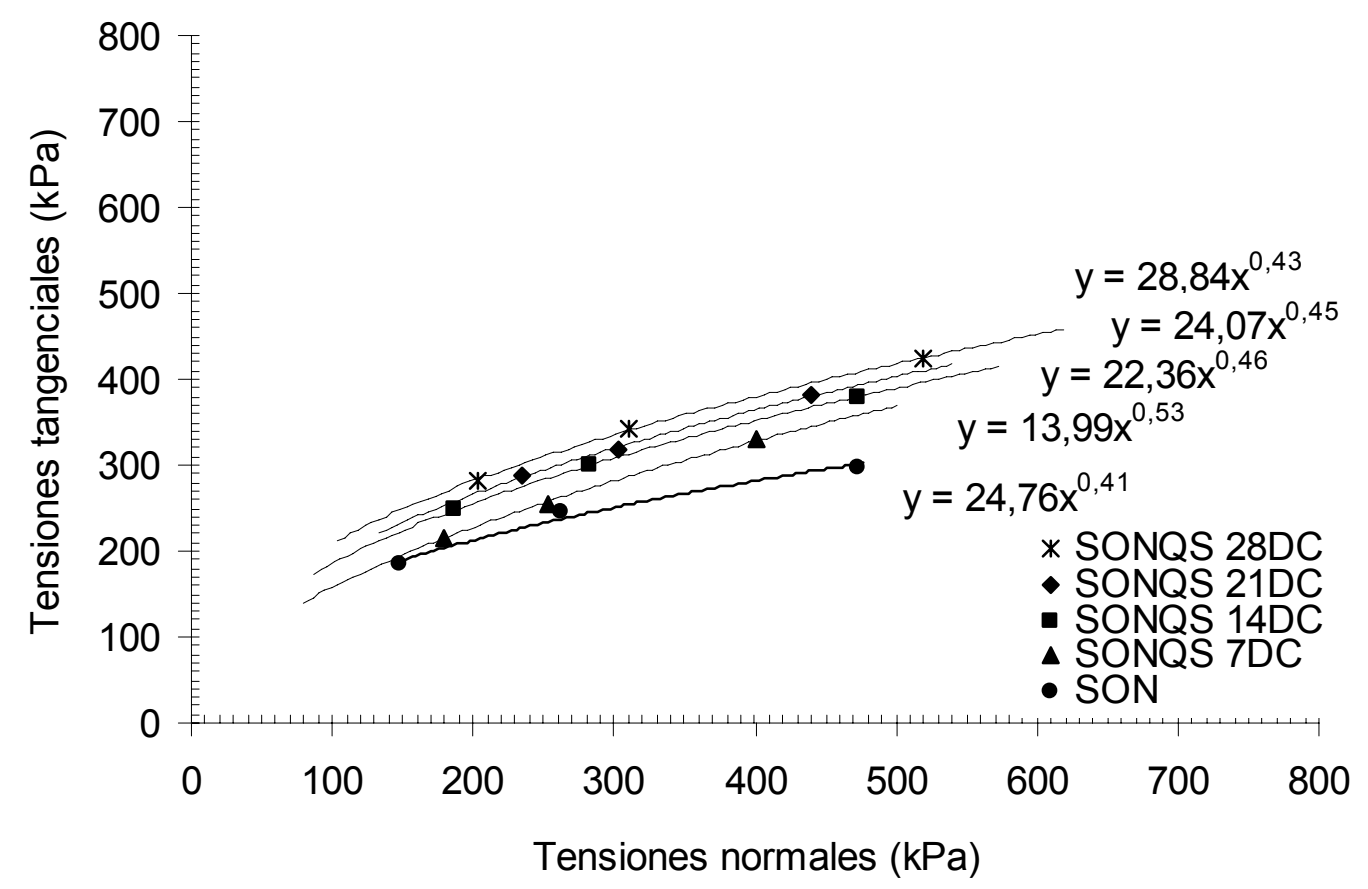

Fig. 3: Envolventes de Mohr para cada periodo de curado - SONQS y SON. 


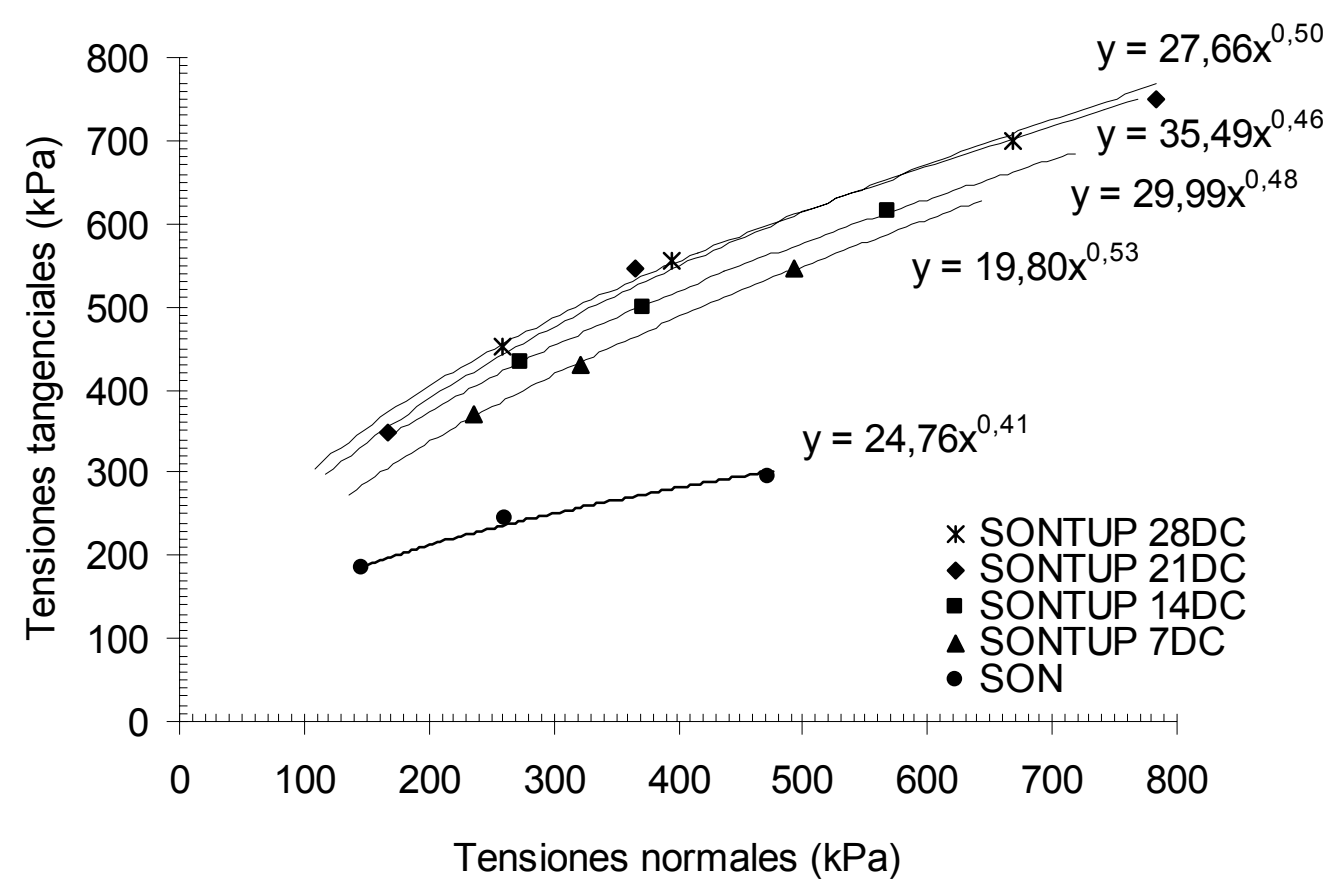

Fig. 4: Envolventes de Mohr para cada periodo de curado - SONTUP y SON.

Consideraciones sobre las distintas resinas aplicadas

Finalmente, se exponen todas las envolventes halladas para 28 DC (Figura 5), con el objeto de evaluar la incidencia que cada resina utilizada tiene sobre los resultados obtenidos. (No se muestran las figuras correspondientes a 7 DC, 14 DC y 21 DC para no redundar en un análisis repetitivo).

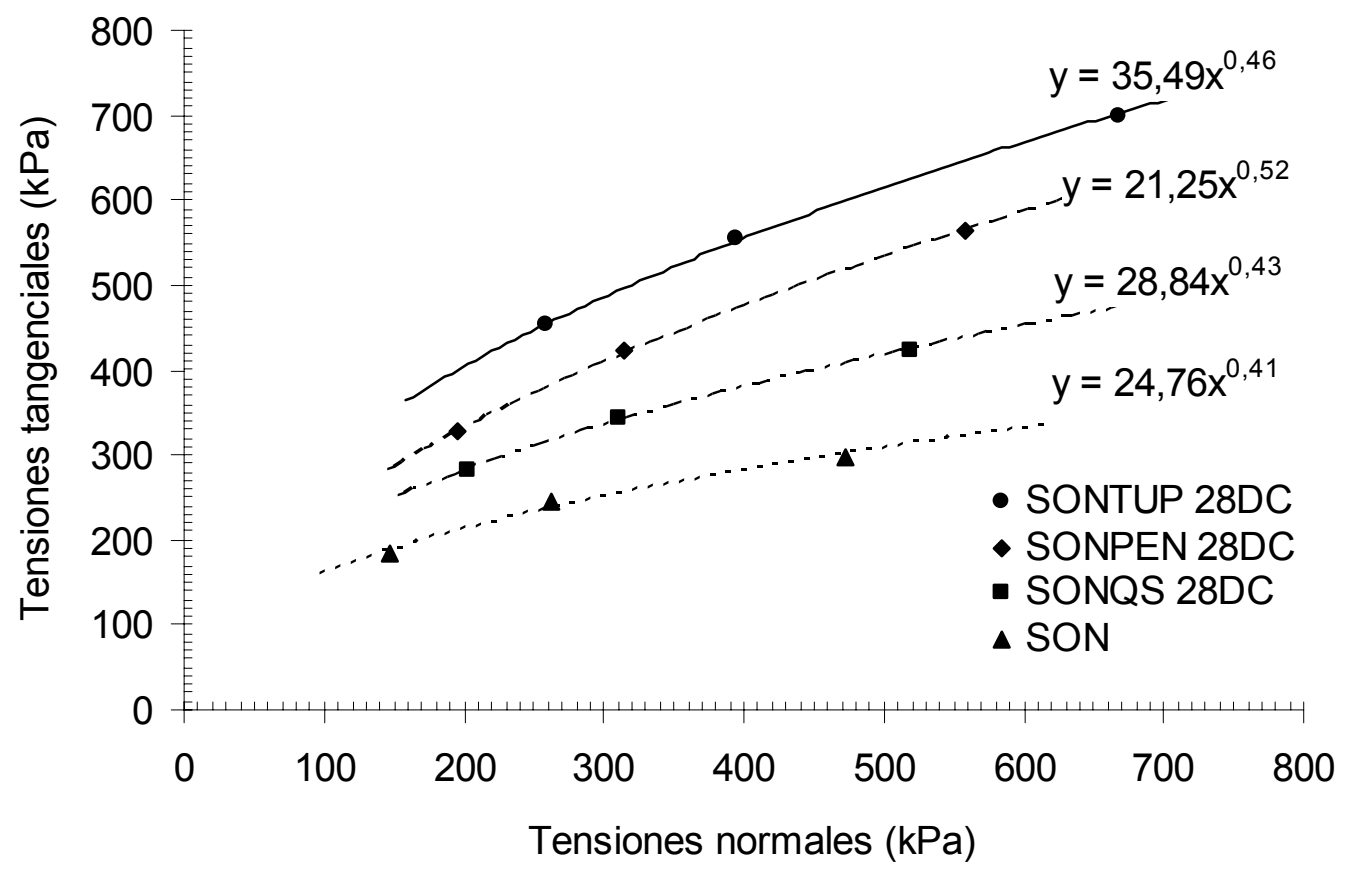

Fig. 5: Envolventes de Mohr - 28 DC.

De la observación de la figura se deduce una mejor respuesta mecánica según el siguiente orden creciente: SONQS, SONPEN, SONTUP. En ella puede observarse que en todos los casos las envolventes del suelo tratado se desarrollan por sobre las del suelo sin tratar, prueba del mejor 
comportamiento mecánico alcanzado. El mismo razonamiento se ha hecho para menores tiempos de curado.

\section{CONCLUSIONES}

En todos los casos estudiados se puede observar la mejora mecánica del suelo natural a partir de la estabilización con cualquiera de las resinas utilizadas, constituidas por tanino, formol y agua.

La resina que mejor comportamiento ha mostrado es la preparada a base de TUPAFIN. La menos eficiente ha sido la que utiliza el producto QS. Entre ellas se ubica la compuesta por PEN (Polvo de Extracto Natural), con valores cercanos a la primera.

Los tiempos de curado crecientes permiten lograr mejores resultados en todos los casos, pero son más evidentes para las mezclas SONTUP y SONPEN.

El producto estabilizador PEN (Polvo de Extracto Natural) agregado al suelo natural, por provenir de la parte inicial del proceso de elaboración del producto final, llamado TUPAFIN, tiene una ventaja económica.

Todos estos resultados permiten inferir la posibilidad de utilización del material estabilizado en aplicaciones que exceden al campo vial, citándose como ejemplo la fabricación de componentes constructivos (bloques, ladrillos, tejuelas, baldosas) para viviendas no tradicionales.

\section{AGRADECIMIENTOS}

Las autoras expresan su reconocimiento al Ing. Héctor Roque Di Rado y al Geol. Adolfo Gustin, por el continuo apoyo ofrecido durante el desarrollo de este trabajo.

\section{REFERENCIAS}

ASTM D2216-05, Standard Test Methods for Laboratory Determination of Water (Moisture) Content of Soil and Rock by Mass, 1-7, West Conshohocken, Pennsylvania, USA (2005).

ASTM D4318-05 - Test Methods for Liquid Limit, Plastic Limit, and Plasticity Index of Soils, 1-16, West Conshohocken, Pennsylvania, USA (2005).

ASTM D1140-00, Standard Test Methods for Amount of Material in Soils Finer than $\mathrm{N}^{\circ} 200(75-\mu \mathrm{m})$ sieve, 1-4, West Conshohocken, Pennsylvania, USA (2006).

ASTM D2487 - 06e1- Standard Practice for Classification of Soils for Engineering Purposes (Unified Soil Classification System), 1-11, West Conshohocken, Pennsylvania, USA (2006).

ASTM D2850 - 03a - Standard Test Method for Unconsolidated-Undrained Triaxial Compression Test on Cohesive Soils, 1-6, West Conshohocken, Pennsylvania, USA (2007).

ASTM D421 - 85, Standard Practice for Dry Preparation of Soil Samples for Particle-Size Analysis and Determination of Soil Constants, 1-2, West Conshohocken, Pennsylvania, USA (2007).

Attom, M.F. y M.M. Al-Sharif; Soil stabilization with burned olive waste, Applied Clay Science: 13(3), 219-230 (1998).

Camacho T.J.F., O.J. Reyes, C. Mayorga y D. F. Méndez; Evaluación de aditivos usados en el tratamiento de arcillas expansivas, Ciencia e Ingeniería Neogranadina: 16, 45-53 (2006).

Chaosheng Tang, y otros cuatro autores; Strength and mechanical behavior of short polypropylene fiber reinforced and cement stabilized clayey soil, Geotextiles and Geomembranes: 25, 194-20 (2007). 
Di Rado H.R., V.E. Fabre y F.D. Miño; Estabilización de Suelos con Tanino, Revista Internacional Información Tecnológica: 12(2), 7-14 (2001).

Di Rado H.R., V.E. Fabre y F.D. Miño, Estabilización de Suelos con Tanino (especialmente en suelos orgánicos), Revista de la Asociación Argentina de Geología Aplicada a la Ingeniería: 18, 28-36 (2002).

Di Rado H.R., V.E. Fabre y R.E. Solari; Universidad Nacional del Noreste, Procedimiento para la estabilización de suelos naturales y composición de aplicación en el mismo, Argentina AR022678B1, Patente Definitiva, 29 de noviembre (2006).

Garnica Anguas P., A. Pérez Zalazar, J.A. Gomez Lopez y E.Y. Obil Vieza; Estabilización de Suelos con Cloruro de Sodio para su uso en las Vías Terrestres, Publicación Técnica, 201, Sanfadila, Querétaro, México (2002).

Gopal R., J. Singh y G. Das; Chemical Stabilisation of Sand: Part VII Natural Resins as Dune Sand Stabiliser, Defense Science Journal: 2, 169-175, (1988).

Head, K.H., Manual of Soil Laboratory Testing, $1^{\text {a }}$ edición,1, p.p. 61-66, Pentech Press, Londres, Inglaterra (1986). 